\title{
Can hepatocellular carcinoma recurrence be prevented after liver transplantation?
}

\author{
Jong Man Kim \\ Department of Surgery, Samsung Medical Center, Sungkyunkwan University School of Medicine, Seoul, Korea
}

Keywords: Immunosuppression; Hepatocellular carcinoma; Liver transplantation

See Article on Page 589

Liver transplantation (LT) is the best treatment strategy for patients with small hepatocellular carcinoma (HCC). However, HCC patients who have undergone multiple locoregional treatments due to high tumor volume or multiple tumors receive LT. ${ }^{1}$ HCC recurrence is the main problem that affects post-transplantation outcomes, with HCC recurrence rates of approximately $10-20 \%{ }^{2}$ The prognosis in liver recipients with recurrent HCC is poor, with a median survival of about 1 year after the diagnosis of recurrent HCC and the majority (55\%) of cases involving extrahepatic recurrence. ${ }^{3}$ Although HCC recurrence remains a challenge for posttransplant survival, adjuvant therapies are not recommended during the post-transplant period.

Calcineurin inhibitors (CNIs) are considered the cornerstone therapeutics that can prevent acute or chronic rejection and help maintain liver graft function after LT. CNIs have a cancer-promoting effect that is associated with their concentrations in the blood. ${ }^{4}$ Due to CNIs, recurrent HCC in liver recipients has been associated with worse prognosis due to rapid progression of $\mathrm{HCC}^{3}$
Kang et al. ${ }^{5}$ compared the time to recurrence (TTR) and overall survival (OS) between two groups of patients who received LT for HCC: the everolimus (EVR) and CNIs group and the CNI-based immunosuppressants (non-EVR) group. Their study also aimed to identify the risk factors for TTR and OS. ${ }^{5}$ Before and after propensity score matching, the TTR and OS of the EVR group were longer than those of the non-EVR group, although the EVR group had a greater number of tumors and a higher frequency of microvascular compared to the non-EVR group. In addition, Kang et al. ${ }^{5}$ divided the EVR group and the non-EVR group into low-risk and high-risk patients based on microvascular invasion and the Milan criteria. In patients without microvascular invasion or within the Milan criteria, the EVR group had longer TTR and OS compared to the non-EVR group, and these values were associated with good prognosis. Interestingly, in patients with microvascular invasion or beyond the Milan criteria, the TTR did not differ between the two groups; however, the OS of the EVR group was longer than that of the non-EVR group. These results showed that, even if HCC recurrence is developed in liver trasnplant recipients, the use of everolimus suppresses cancer progression. Finally, the use of everolimus-based immunosuppression was a significant factor for

\begin{abstract}
Abbreviations:
AFP, alpha-fetoprotein; CNI, calcineurin inhibitor; EVR, everolimus; HCC, hepatocellular carcinoma; LT, liver transplantation; mTORi, mammalian target of rapamycin inhibitors; OS, overall survival; RFS, recurrence-free survival; SiLVER, Sirolimus in Liver Transplant Recipients with HCC study; TTR, time to recurrence
\end{abstract}

\section{Corresponding author : Jong Man Kim}

Department of Surgery, Samsung Medical Center, Sungkyunkwan University School of Medicine, 81 Irwon-ro, Gangnam-gu, Seoul 06351, Korea

Tel: +82-2-3410-1719, Fax: +82-2-3410-0040

E-mail:yjongman21@gmail.com

https://orcid.org/0000-0002-1903-8354 
longer TTR and OS in HCC patients after LT. The side effects or complications of everolimus were different from those of $\mathrm{CNIs}^{6}{ }^{6}$ Kang et al. ${ }^{5}$ also revealed that the number of patients with dyslipidemia and proteinuria were significantly higher in the EVR group than in the non-EVR group. However, there was no difference in the frequency of acute rejection, biliary complications, portal vein thrombosis, wound infection, mouth ulcers, or renal failure between the two groups.

The mammalian target of rapamycin inhibitors (mTORi), such as everolimus or sirolimus, represent alternative immunosuppressive agents, and previous studies has confirmed the antineoplastic effect of mTORi. ${ }^{6,7}$ The Sirolimus in Liver Transplant Recipients with HCC study (SiLVER) trial, a large, prospective, randomized trial that enrolled 525 liver transplant recipients with HCC, compared the recurrence-free survival (RFS) and OS between sirolimusbased and sirolimus-free immunosuppression therapies. Although the primary endpoint of improved RFS with sirolimus was not met, better RFS and OS were noted for patients in the sirolimus-treatment arm in the first 3 to 5 years. ${ }^{8}$ However, the differences in RFS and OS between the two groups were not statistically significant after 8 years. Everolimus data from two randomized liver transplant trials ( $\mathrm{H} 2304$ and H2307) showed that the frequency of $\mathrm{HCC}$ recurrence was lower in the combination of everolimus and reduced tacrolimus group (5.9\% [1 of 17] vs. 23.1\% [6 of 26], $P=0.215$ ) in patients beyond the Milan criteria compared to that of patients within the Milan criteria (2.9\% [3 of 102] vs. $2.1 \%$ [2 of 96$], P=1.000$ ), irrespective of the pre-transplant alpha-fetoprotein (AFP) level; however, this result was not statistically significant. $^{9}$ A recent study which used the SiLVER-trial data demonstrated that sirolimus treatment is beneficial when given more than 3 months after LT for HCC and is advantageous particularly for patients within the Milan criteria with an elevated AFP $>10$ $\mathrm{ng} / \mathrm{mL} .^{10}$

In summary, $\mathrm{HCC}$ recurrence is a very important factor that contributes to patient survival. Based on Kang et al.'s study, ${ }^{5}$ everolimus may inhibit $\mathrm{HCC}$ recurrence and increase OS by suppressing progression even in recurrent HCC. The addition of everolimus to the CNIs-based immunosuppressive regimen can be considered for patients with HCC even though it has been associated with several adverse events, such as dyslipidemia and proteinuria. However, since this study was retrospective, it will be important to identify the patients who can benefit from everolimus therapy based on the risk factors for $\mathrm{HCC}$ recurrence.

\section{Conflicts of Interest}

The author has no conflicts to disclose.

\section{REFERENCES}

1. Kim JM, Kim DG, Kim J, Lee K, Lee KW, Ryu JH, et al. Outcomes after liver transplantation in Korea: incidence and risk factors from Korean transplantation registry. Clin Mol Hepatol 2021;27:451-462.

2. Fernandez-Sevilla E, Allard MA, Selten J, Golse N, Vibert E, Sa Cunha $A$, et al. Recurrence of hepatocellular carcinoma after liver transplantation: is there a place for resection? Liver Transpl 2017;23:440-447.

3. Kim M, Rhu J, Choi GS, Kim JM, Joh JW. Risk factors for poor survival after recurrence of hepatocellular carcinoma after liver transplantation. Ann Surg Treat Res 2021;101:28-36.

4. Bhat M, Mara K, Dierkhising R, Watt KDS. Immunosuppression, race, and donor-related risk factors affect de novo cancer incidence across solid organ transplant recipients. Mayo Clin Proc 2018;93: 1236-1246.

5. Kang I, Lee JG, Choi SH, Kim HJ, Han DH, Choi GH, et al. Impact of everolimus on survival after liver transplantation for hepatocellular carcinoma. Clin Mol Hepatol 2021;27:589-602.

6. Yee ML, Tan HH. Use of everolimus in liver transplantation. World J Hepatol 2017;9:990-1000.

7. Toso C, Merani S, Bigam DL, Shapiro AM, Kneteman NM. Sirolimusbased immunosuppression is associated with increased survival after liver transplantation for hepatocellular carcinoma. Hepatology 2010;51:1237-1243.

8. Geissler EK, Schnitzbauer AA, Zülke C, Lamby PE, Proneth A, Duvoux $C$, et al. Sirolimus use in liver transplant recipients with hepatocellular carcinoma: a randomized, multicenter, open-label phase 3 trial. Transplantation 2016;100:116-125.

9. Lee SG, Jeng LB, Saliba F, Singh Soin A, Lee WC, De Simone P, et al. Efficacy and safety of everolimus with reduced tacrolimus in liver transplant recipients: 24-month results from the pooled analysis of 2 randomized controlled trials. Transplantation 2021;105:1564-1575.

10. Schnitzbauer AA, Filmann N, Adam R, Bachellier P, Bechstein WO, Becker $T$, et al. mTOR inhibition is most beneficial after liver transplantation for hepatocellular carcinoma in patients with active tumors. Ann Surg 2020;272:855-862. 